\title{
A Novel Polarization-Independent, Free-Space, Microwave Beam Splitter Utilizing an Inkjet-Printed, 2-D Array Frequency Selective Surface
}

\author{
James R. Cooper, Sangkil Kim, and Manos M. Tentzeris, Fellow, IEEE
}

\begin{abstract}
This letter introduces the first beam splitter that acts on free-space, planar, microwave frequency waves below $80 \mathrm{GHz}$. The design utilizes a frequency selective surface (FSS) operating away from its normal frequency of operation. The FSS is constructed from an array of scalable printed patterns, which allows for the tenability of the FSS to any frequency that the fabrication method will allow. The basic operation of an FSS, the reasoning behind selecting the chosen unit element and fabrication, and a comparison of simulated and measured results are discussed in detail. An inkjet-printed prototype on a glossy paper substrate utilizing silver nanoparticles verifies the easy and scalable low-cost fabrication capability of the proposed topology.
\end{abstract}

Index Terms-Beam splitting, frequency selective surfaces (FSSs), inkjet printing, microwave devices, printed circuits.

\section{INTRODUCTION}

\section{A. Beam Splitting}

\begin{abstract}
A BEAM splitter, which is traditionally used in optics, takes a propagating electromagnetic wave and splits it into multiple waves. This splitting may be a polarizing split, which separates the wave into two waves with orthogonal polarizations, like polarized lenses; frequency split, which splits the wave by frequency spectrum, such as a prism; or amplitude split, which splits the wave into multiple waves with a set power ratio, such as a semi-reflective mirror. A free-space beam splitter acts on a TEM wave traveling in open air or a vacuum. Free-space amplitude beam splitters have been around for many years [1], [2] and are commonly used in interferometers and laser output-power trackers, but have mostly been confined to optical frequencies. This is due to beam splitters being based on total internal refraction in between two medium or on semi-transparent mirrors. The first relies on the space between the medium being dependant on wavelength. The second relies on the skin depth of the metal in the mirror being very small. Both become very impractical for millimeter-wave and lower frequencies. However, due to recent advances in wireless power transfer and ad hoc RF networks, it is desired that similar devices for lower frequencies be developed.
\end{abstract}

Manuscript received April 03, 2012; revised May 28, 2012; accepted May 28, 2012. Date of publication June 13, 2012; date of current version June 27 2012. This work was supported by of an NSF Graduate Fellowship, the NSF under Grant ECS-0801798, and the IFC/SRC.

The authors are with the Department of Electrical and Computer Engineering, Georgia Institute of Technology, Atlanta, GA 30332 USA (e-mail: JCooper45@gatech.edu).

Color versions of one or more of the figures in this letter are available online at http://ieeexplore.ieee.org.

Digital Object Identifier 10.1109/LAWP.2012.2204715

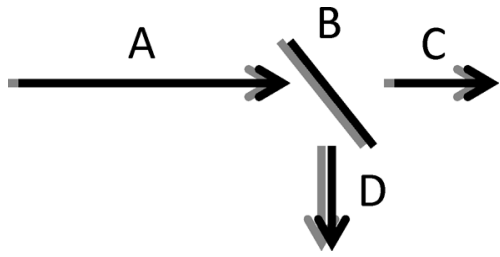

Fig. 1. Proposed free-space power splitter. A power splitter (B) takes an incoming incident wave (A) and splits it into two parts: a through transmitted signal (C) and a reference signal (D).

A free-space amplitude beam splitter for $80-110 \mathrm{GHz}$ [3] has been developed. This beam splitter is based on a metamaterial constructed by placing a metal grating on a dielectric slab, where the slab depth has a linear ratio with the wavelength of the desired frequency of operation. This increases the cost of fabrication, decreases the ease of fabrication, and leads the device to become bulky at lower frequencies. A spatial beam splitter has been developed for submillimeter-wave applications $(7 \mathrm{GHz}$ in this application, but scalable to a wider range) [4]. However, this design requires a single transmission line feed and does not split free-space waves. Like [3], it also utilizes dielectric slab-based metamaterial, which adds cost and difficulty in fabrication.

In this letter, a polarization-independent, free-space amplitude beam splitter is presented, which operates in the submillimeter-wave frequency range. It is based on printed frequency selective surfaces (FSSs), which are a cheaply fabricated and easily scaled metamaterial. It acts on a single plane wave, which it splits into two parts with pointing vectors $90^{\circ}$ apart, as shown in Fig. 1. This letter will cover the case where it is desired to split the wave into two equal parts. However, as will be shown, a wide range of split ratios can be achieved by adjusting the dimensions of the FSS unit cell.

\section{B. Frequency Selective Surfaces}

Frequency selective surfaces, which are a type of metamaterial, have been studied for a long time due to their many properties. Their frequency selectivity makes them useful in multiband reflector antennas [5] due to the fact that for carefully chosen dimensions and spacing, they can act as magnetically conductive surfaces that are used as reflectors for dipoles and other compact antennas [6] to increase directivity. Because of their appearance as a high-impedance surface, and thus ability to suppress leaky surface traveling waves, they have been used in patch antennas [7], [8] to increase efficiency. Due to their band-gap properties, they have been used in leaky resonant cavity antennas [9].

Frequency selective surfaces are normally composed of some type of periodic structure. This could be a 2-D array of 

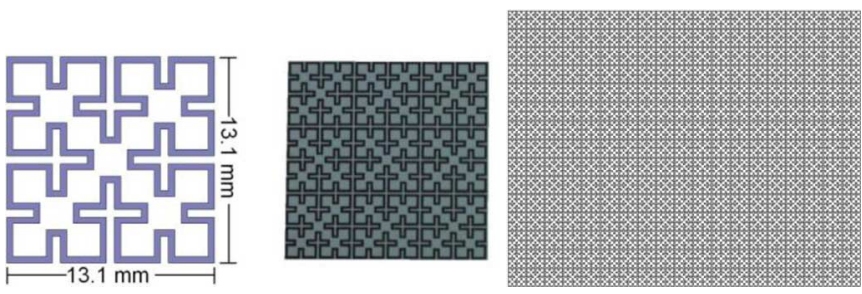

Fig. 2. Third-generation convoluted squares used for the FSS. (left) Bitmap image of the unit cell that was used for simulation and printing. (center) Photograph of a $3 \times 3$ array of the cells after printing. (right) Full schematic of one of the sheets. The cells are spaced $0.5 \mathrm{~mm}$ apart.

dipoles [10], an array of loops linked together with lumped elements [11], stacked arrays of dielectric rods [12], etc. In any configuration, they have the unique property that at a single frequency they act as an infinite impedance surface to plane waves, and at other frequencies they act as transparent surfaces [6]. This means that there exists a point, somewhere between the passand stopband, that the FSS allows half the power to pass through and reflects the other half.

The 2-D FSS has been shown to be fully operational when working at a $45^{\circ}$ incidence, as long as the elements and their spacing are small [13]. If the FSS is illuminated at the halfpower frequency point, a frequency located between the point of transparency and full reflection, at an angle of incidence $45^{\circ}$, then the result would be what is shown, and desired, in Fig. 1.

Section II will cover how the FSS structure was chosen and the manufacturing proses chosen for fabrication. Section III will cover how the measurements were set up and made. Section IV will cover the results and create a clear definition for how to measure performance of submillimeter-wave frequency beam splitters. Section V will bring together the results and discuss possible applications for the beam splitter.

\section{DeSIGn AND FABRICATION}

\section{A. Unit Cell}

For ease of fabrication and ease of scaling, the FSS was designed using a single printable layer of a 2-D array of unit cells. The cells were chosen on the criteria of the surface being able to operate at $45^{\circ}$ of incidence to the transmitted signal, and needing to be nonpolarized to make the surface operate the same independent of polarization of incident wave. According to [5], in order to avoid grating issues, which causes shifts in the center frequency of the surface, the unit cells should be electrically small, on the order of $<1 / 8$ of a wavelength. Most unit cells, such as the tripole, are close to $1 / 2$ wavelength in size. Therefore, a convolutable shape, or shape that can be made to minimize area while maintaining a large diameter, was chosen [10]. The Hilbert curve allows itself to be made exceptionally small, but it is polarized. Therefore, the convoluted square, which can be seen in the left of Fig. 2, was chosen for this design since it features the smallest nonpolarized shape.

The total number of cells in the 2-D array was chosen so that at $45^{\circ}$ of incidence, the cross-sectional area of the FSS would be roughly the size of the plane-wave front.

\section{B. Inkjet Printing}

Inkjet printing was chosen to fabricate the FSS prototype due to its rapidness of fabrication and potential for low cost. It was printed using silver nanoparticles in a solvent. Several layers

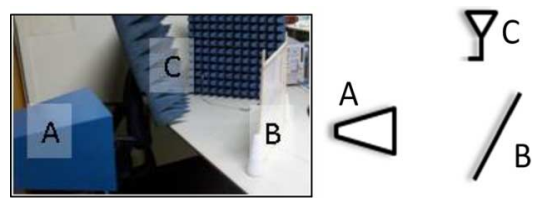

Fig. 3. Measurement setup for the power splitter. A horn antenna (A) illuminates a monopole antenna (C), first directly, then through an angle FSS (B), then at a $90^{\circ}$ angle reflected off the FSS.

were printed in order to maximize the conductivity. The printing was placed in an oven so as to evaporate the solvent and fuse the silver particles together forming a continuous conductive structure. It was printed on 9-mil-thick glossy paper, with a dielectric constant of about 3.0 and a loss tangent of 0.07 .

Due to splatter of the ink, a minimum dimension of $400 \mu \mathrm{m}$ was chosen. This limited the design to a third-generation convoluted square with line widths of $400 \mu \mathrm{m}$ that allowed for a sufficiently small unit-cell size, which could not be achieved with a second-generation unit cell, while keeping the line width and interline spacing over $400 \mu \mathrm{m}$, which could not be achieved with a fourth-generation unit cell. Due to the chosen rejection-band frequency of $3 \mathrm{GHz}$ (before the effects of the glossy paper), this gave a unit-cell dimension of $13.1 \times 13.1 \mathrm{~mm}^{2}$. This band was chosen so as to place the lower end of the power split-band at $2.4 \mathrm{GHz}$. Four sheets, each with a $19 \times 15$ array of the unit elements, were printed. The sheets were attached together in a $2 \times 2$ array with spacing to keep consistent with the spacing between the individual elements. Four sheets were chosen so as to guarantee that the surface would be large enough to completely block the transmitted signal from making it to the receiver, while at the same time keeping the total surface small enough to be easily set up and moved around. A bitmap of the unit element, along with a photograph of a $3 \times 3$ section of the fabricated FSS and a scale schematic of the FSS, can be seen in Fig. 2.

\section{Measurement SetuP}

Fig. 3 shows the setup used for testing the FSS. A horn antenna with a gain of $25 \mathrm{dBi}$ was used to illuminate the FSS, and two receiving monopole antennas were used to measure the power levels of the split signals. The horn antenna was chosen so that the FSS could be kept out of the near field of the antenna while still acting as a complete barrier between the transmit and receive antennas. The horn and monopoles were chosen such that they each had a bandwidth wide enough to cover the desired frequency band of the FSS. For measurement, the horn was rotated around the FSS, keeping an equal distance. Both receiving antennas were also placed at equal distance from the surface and $90^{\circ}$ apart. The value of transmission for both the through transmission (line $\mathrm{C}$ in Fig. 1), and the value of reflection (line $\mathrm{D}$ in Fig. 1) were normalized to the value of direct radiation from the horn to the monopoles.

\section{Results AND BANDwidth Definition}

\section{A. Results}

Fig. 4 shows a comparison between the simulated results, which were made using Ansoft HFSS, and the measured results. The through and reflected (reference) are defined in Fig. 1. As can be seen, the surface acts as a good FSS and does a good job of splitting the wave. The simulated reflected and through 


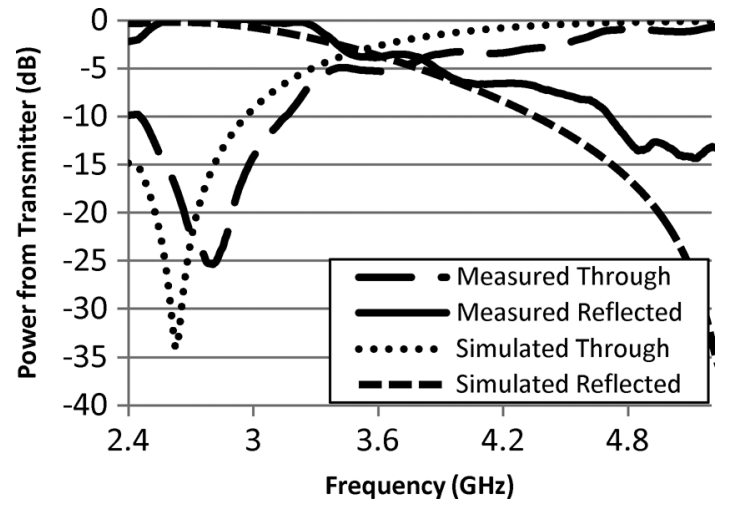

Fig. 4. Power levels received after passing through the power splitter. This plot shows a comparison between the power sent through the FSS and the power reflected off the FSS.

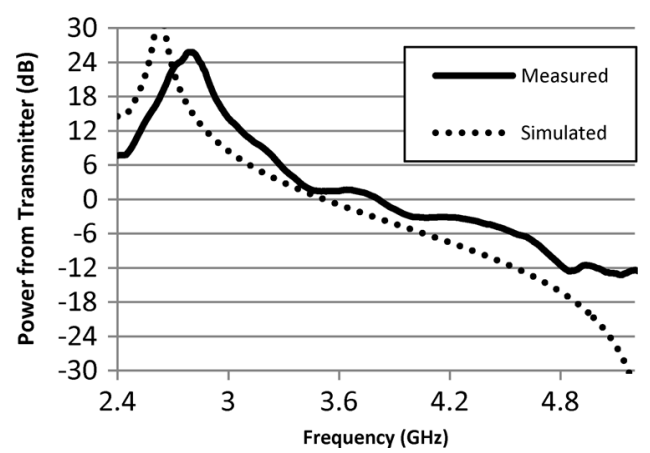

Fig. 5. Power ratio between transmitted and reflected power. This plot shows the power ratios between the power sent through the FSS and the power reflected off the FSS. As can be seen, the FSS has a 2:1-to-1:2 ratio bandwidth of $10 \mathrm{MHz}$.

cross at $-3.1 \mathrm{~dB}$ at $3.5 \mathrm{GHz}$. The measured through and reflected cross at $-4 \mathrm{~dB}$ at $3.8 \mathrm{GHz}$. The loss of $1 \mathrm{~dB}$ and the frequency shift could be the result of misalignment between the different printed sheets, effects of the wooden dowels used to hold the surface steady, effects of the adhesive used, or bends in the surface. These could be fixed by attaching the FSS to a porous Styrofoam sheet, which is cheap and has a dielectric constant very close to air, and by printing the FSS on a single continuous sheet with a larger inkjet printer.

\section{B. Operational Bandwidth}

Reference [3] defined the operational bandwidth to be when the through and reflected signals were within $0.8 \mathrm{~dB}$ of each. This definition was chosen arbitrarily based upon how that specific beam splitter operated. Following suit, a new definition is chosen in this letter. Here, the bandwidth will be chosen to be the range in which the ratio between reflected and through signal varies between $2: 1$ and $1: 2$, or 3 to $-3 \mathrm{~dB}$. As can be seen in Fig. 5, the simulated bandwidth comes out to be $500 \mathrm{MHz}$, or $14 \%$ bandwidth. As is to be expected from the losses observed in the system, the measured bandwidth is larger at $620 \mathrm{MHz}$, or $16 \%$.

\section{Splitting Ratios}

Thus far, this letter has focused on the design on an amplitude beam splitter, which equally splits a beam in two directions. However, it is often desired for the split to be uneven, such as in a laser output power tracker. In this situation, it is desired that most of the power be transmitted in a single direction and very little be used for tracking purposes. The presented beam splitter has the ability to split at ratios other than $1: 1$. As can be seen in Fig. 5, a split ratio can be achieved from $0(1: 1)$ to $25 \mathrm{~dB}(316: 1)$ with the measured FSS. From the simulation, it appears that the theoretical limit to the split ratio is much higher.

\section{CONCLUSION}

Hereto has been presented the first nonpolarizing amplitude beam splitter without surface-mount components that operates at low microwave frequencies with performance verified by an inkjet-printed prototype on a glossy paper substrate designed around $3 \mathrm{GHz}$. It has been shown, through simulation and measurement, that over a narrow bandwidth, this beam splitter can split an incident beam into two equal parts traveling in directions $90^{\circ}$ apart with less than $1 \mathrm{~dB}$ of attenuation. It has also been shown that a splitting ratio other than 1:1 can be achieved by scaling the unit elements of the material.

As seen in [13], a wide range of angles for the reflected beam can be achieved. This means that this device could be implemented in ad hoc wireless networks, where it is necessary to disperse a signal in multiple directions in order to illuminate multiple receivers. Utilizing the various splitting ratios discussed in Section IV-C, the beam splitter could also be used in wireless power transfer applications as part of a power tracker to measure how much power is being transmitted from a source. Due to the strong frequency response, the beam splitter could be used in spectrum analysis, where multiple surfaces can be used to direct a signal toward multiple sensors.

\section{REFERENCES}

[1] C. Thomas and D. C. Smith, "An automatic recording spectrophometer," Rev. Sci. Instrum., vol. 18, no. 3, pp. 173-181, 1947.

[2] F. Back, "Lens tester for photographic lenses," Rev. Sci. Instrum., vol. 21 , no. 8, pp. 722-724, 1950 .

[3] R. Watanabe, "A novel polarization-independent beam splitter," IEEE Trans. Microw. Theory Tech., vol. 28, no. 7, pp. 685-689, Jul. 1980.

[4] R.-B. Hwang, N.-C. Hsu, and C.-Y. Chin, "A spatial beam splitter consisting of a near-zero refractive index medium," IEEE Trans. Antennas Propag., vol. 60, no. 1, pp. 417-420, Jan. 2012.

[5] E. A. Parker and S. M. A. Hamdy, "Rings as elements for frequency selective surfaces," Electron. Lett., vol. 17, no. 17, pp. 612-614, 1981.

[6] R. C. Hansen, "Effects of a high-impedance screen on a dipole antenna," IEEE Antennas Wireless Propog. Lett., vol. 1, pp. 46-49, 2002.

[7] R. Gonzalo, P. De Maagt, and M. Sorolla, "Enhanced patch-antenna performance by suppressing surface waves using photonic-bandgap substrates," IEEE Trans. Microw. Theory Tech., vol. 47, no. 11, pp. 2131-2138, Nov. 1999

[8] D. Sievenpiper, L. Zhang, and R. F. J. Broas, "High-impedance electromagnetic surfaces with a forbidden frequency band," IEEE Trans. Microw. Theory Tech., vol. 47, no. 11, pp. 2059-2074, Nov. 1999.

[9] A. Foroozesh and L. Shafai, "On the design of high-gain resonant cavity antennas using different highly-reflective frequency selective surfaces as the superstrates," in Proc. IEEE Antennas Propog. Soc. Int. Symp., 2010, pp. 1-4.

[10] E. A. Parker and A. N. A. El Sheikh, "Convoluted array elements and reduced size unit cells for frequency-selective surfaces," Proc. Inst. Elect. Eng. Part H, Microw., Antennas Propog., vol. 138, no. 1, pp. 19-22, 1991.

[11] F. Bayatpur and K. Sarabandi, "Single-layer high-order miniaturizedelement frequency-selective surfaces," IEEE Trans. Microw. Theory Tech., vol. 56, no. 4, pp. 774-781, Apr. 2008.

[12] C. Biancotto and P. Record, "Triangular lattice dielectric EBG antenna," IEEE Antennas Wireless Propag. Lett., vol. 9, pp. 95-98, 2010

[13] G. Kiani, K. Ford, K. Esselle, and A. Weily, "Oblique incidence performance of an active square loop frequency selective surface," in Proc. 2nd EuCAP, 2007, pp. 1-4. 